\title{
Tilings, Quasicrystals, Discrete Planes, Generalized Substitutions, and Multidimensional Continued Fractions
}

\author{
Pierre Arnoux, ${ }^{1}$ Valérie Berthé, ${ }^{1 \dagger}$ Hiromi Ei ${ }^{2}$ and Shunji Ito ${ }^{2}$ \\ ${ }^{1}$ Institut de Mathématiques de Luminy (UPR 9016), 163 Avenue de Luminy, case 90713288 Marseille Cedex 9, \\ France \\ ${ }^{2}$ Department of Mathematics, Tsuda College, Tsuda-Machi, Kodaira, Tokyo187, Japan
}

received February 20, 2001, revised May 4, 2001, accepted May 20, 2001.

The aim of this paper is to give an overview of recent results about tilings, discrete approximations of lines and planes, and Markov partitions for toral automorphisms. The main tool is a generalization of the notion of substitution. The simplest examples which correspond to algebraic parameters, are related to the iteration of one substitution, but we show that it is possible to treat arbitrary irrational examples by using multidimensional continued fractions. We give some non-trivial applications to Diophantine approximation, numeration systems and tilings, and we expose the main unsolved questions.

Keywords: Substitutions, translations on compact groups, tilings, atomic surface, fractal sets, Markov partitions, numeration systems.

\section{Introduction}

In this paper, we survey a number of recent results relating self-similar tilings in two or more dimensions, Diophantine approximation and continued fractions in the multidimensional case, discrete approximations of planes, $\beta$-expansions for some algebraic integers and their natural extensions, and Markov partitions for hyperbolic toral automorphisms.

The main tool for these results is a notion of generalized substitution, that is, a geometric extension to discrete segments or $k$-dimensional faces in dimension $d$ of substitutions defined over $d$-letter alphabets; we introduce this notion in more details in Section 4.

We will first, in Section 2, recall what is known in dimension 1; in that case, our knowledge is very complete (because the situation is very simple!), and it can serve as a guideline. We would like to obtain similar results in higher dimension; it is useful, in this situation, to keep in mind the one-dimensional analogue.

\footnotetext{
${ }^{\dagger}$ We would like to thank Tsuda College, whose hospitality contributed to this paper. 
In Section 3, we will work out one of the simplest examples in dimension 2. We will give completely constructive algorithms, and show some surprising facts; in particular, a certain domain with fractal boundary can be obtained by apparently very different constructions, either geometric or arithmetic.

We introduce in Section 4 the notions of atomic surface and stepped surface, and we explain how the experimental findings of Section 3 can be proved. These two notions which can be considered as dual concepts, prove to be useful in several mathematical fields (including fractal geometry, tilings, combinatorics, ergodic theory and spectral analysis, number theory and numeration systems), but also in theoretical computer science and theoretical physics, with the structural properties of quasi-crystals.

In Section 5, we explain how all this can be extended to the non-algebraic case, using generalized continued fractions. In Section 6 we give a recent application of these results.

\section{The one-dimensional case}

\subsection{The Fibonacci tiling}

The simplest example of a self-similar non-periodic tiling is the Fibonacci tiling of the line; it is a tiling by two intervals, interval $a$, of length $\phi=\frac{1+\sqrt{5}}{2}$, and interval $b$ of length 1 , the structure of the tiling being the unique fixed point of the Fibonacci substitution $a \mapsto a b, b \mapsto a$. (Recall that a substitution on the alphabet $\{a, b\}$ is a map $\sigma$ on the set of finite or infinite words on this alphabet endowed with the concatenation, that associates with each word the unique word obtained by replacing the letter $a$ (resp. $b$ ) by a non-empty word $\sigma(a)$ (resp. $\sigma(b)$ ). It is easy to check that the Fibonacci substitution has a unique fixed point, which is an infinite word starting with abaababaabaab....)

If we divide each interval $a$ in an interval $a^{\prime}$ of length 1 , and an interval $b^{\prime}$ of length $1 / \phi$, and rename the interval $b$ as $a^{\prime}$, we obtain a new tiling (called inflation of the initial tiling) which is homothetic to the initial one by an homothety of ratio $1 / \phi$; on the other hand, it is easy to check that each interval $b$ is preceded by an interval $a$; if we merge each pair of consecutive intervals $a, b$ as one interval $a^{\prime \prime}$, of length $\phi^{2}$, and rename the remaining intervals as $b^{\prime \prime}$, we obtain another tiling (deflation of the initial one), homothetic to the initial one by an homothety of ratio $\phi$.

This remarkable tiling has been much studied, starting with the pioneering work of de Bruijn $[15,16]$; it can be obtained in a number of different ways, notably by the "cut and project" method (see for instance [31]), as projection of a discrete approximation of the line through 0 with slope $1 / \phi$ (see Figure 2.1). In particular, one can consider the tilings obtained from it by translation of the origin, and their closure for the natural topology on the space of tilings of the line; this abstract space, if one identifies tilings that coincide out of a bounded set, has a geometric model as a flat torus, endowed with a partition in two rectangles (see Figure 2); the tiling corresponding to the point $p$ appears as the trace, on the geodesic with slope $1 / \phi$ and initial point $p$, of this partition. For more details, see [4].

We see here that two different operations act on this space of tilings: the translation is the linear flow with slope $1 / \phi$, while the inflation corresponds to the automorphism with matrix $\left(\begin{array}{ll}1 & 1 \\ 1 & 0\end{array}\right)$, which preserves the lines with slope $1 / \phi$.

The symbolic sequences associated with these tilings have a nice interpretation: they are symbolic dynamics for the rotation by the golden number on a circle of length 1 . The self-similarity of these sequences has a dynamical meaning: if we induce this rotation on an interval of length $1 / \phi$, we obtain again a rotation, conjugate to the initial one (recall that if $T: X \rightarrow X$ is a dynamical system, the induced 


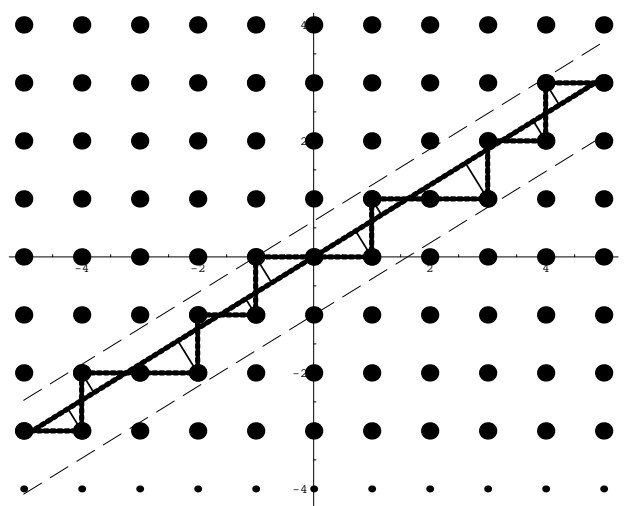

Fig. 1: The Fibonacci tiling by the "cut and project" method

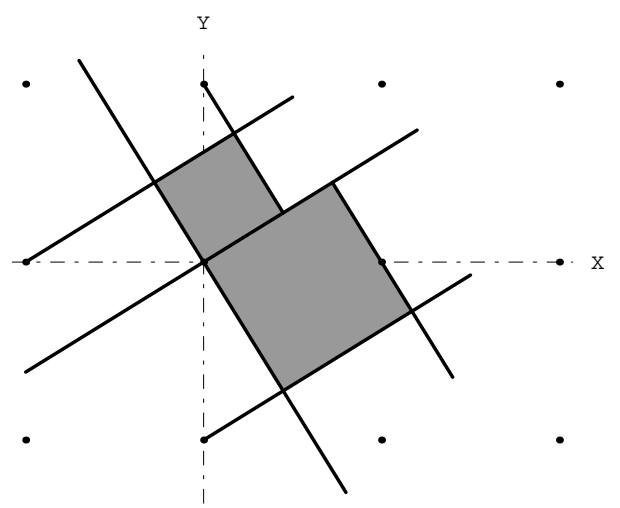

Fig. 2: The space of tilings generated by the Fibonacci tiling

map $T_{\mid A}$ of $T$ on a subset $A \subset X$ is the first return map to $A$, defined on $A$ by $T_{\mid A}(x)=T^{n_{x}}(x)$, where $n_{x}$ is the smallest strictly positive integer $n$ such that $T^{n}(x)$ belongs to $A$ ).

One can also give an arithmetic definition of the Fibonacci tiling: it is easy to prove that each positive real number can be written in a unique way $x=\sum_{n=-N}^{\infty} \varepsilon_{n} \phi^{-n}$, with $\varepsilon_{n}=0$ or 1 , no two consecutive 1 's, and not ending in the periodic sequence $010101 \ldots$; this is the real counterpart of the well-known Zeckendorf expansion [38] of integers in terms of Fibonacci numbers. If we partition the line according to the negative part $\varepsilon_{-N} \ldots \varepsilon_{-1}$ of this expansion, we recover the Fibonacci tiling (the lengths of the tiles are 1 and $1 / \phi$, according to whether $\varepsilon_{-1}$ equals 0 or not).

\subsection{Self-similar quadratic tilings of the line}

Similar tilings can be easily obtained for reduced quadratic integers. The simplest way is to consider a matrix in $S L(2, \mathbb{Z})$; this defines a toral automorphism. Using the classical procedure of Adler-Weiss ([1]), one can obtain a Markov partition for this automorphism, by refining a partition of the torus made of two 
rectangles with sides parallel to the eigendirections of the automorphism.

If one consider a geodesic in the expanding direction, the intersection of this geodesic with the 2 rectangles determines a tiling of the line which is self-similar, the factor of self-similarity being the eigenvalue of the automorphism. Everything works here as in the Fibonacci case.

Some remarks are in order here: first, the slope of the expanding line is a quadratic integer, with a special property: these are exactly the real numbers which have an immediately periodic continued fraction expansion.

Second, the first return map to a transverse circle of the flow along the expanding direction (unstable flow of the automorphism) is a rotation which is conjugate to its induced map on a suitable subinterval.

Third, the tiling we obtain is characterized by two properties: the sizes of the tiles $a, b$ and their combinatorics (the sequence $a b a a \ldots$ ), and it seems that one determines the other. This is not the general case: the size of the tiles determine the small-scale structure of the tiling, while the combinatorics determine the large scale structure, and they are linked in this special case because of the self-similarity. We will come back to this idea in the multidimensional case in Section 5.3.

\subsection{Sturmian tilings}

We can get in this way only a countable family of tiling dynamical systems, linked with the quadratic integers. It is possible to generalize by using the "cut and project" method illustrated in Figure 1, with an arbitrary pair of transverse lines, taking the best discrete approximation of the first line, and projecting along the second line.

The tilings thus obtained have arbitrary ratio for tiles sizes, but their combinatorics are very specific sequences, named Sturmian sequences (see [28]). These are the non-periodic sequences of lowest complexity function, and are obtained as codings of circle rotations with respect to the natural partition of the circle in two intervals, determined by a point and its image by the rotation.

One thus gets a family of tilings which are no more self-similar; one can nevertheless define on these tilings an inflation, by subdividing the largest tile, and a deflation by merging the isolated tile.

This family of tilings has been completely studied (see $[10,26]$ for complete references on Sturmian sequences, and [4] for Sturmian tilings). The global picture is the following: for each pair of slopes of transverse lines, there is a space of tilings, globally invariant by translation, which is isomorphic to a torus if we identify tilings that differ only on a bounded domain. The pair of slopes can be considered as parameters for a family of dynamical systems, and the inflation map acts on this pair of parameters; its action on the second parameter (the projection line) is just the Gauss map associated with the continued fraction expansion, and it is globally a natural extension of this Gauss map.

The best intrinsic way to understand this deflation map is to consider it as a first return map of a flow, by defining a flow on the space of tilings that multiply tile sizes by $e^{t}$, and using inflation to limit the size of the tiles. This flow on the parameter space has a nice geometric interpretation as the geodesic flow on the unit tangent bundle $S L(2, \mathbb{Z}) \backslash S L(2, \mathbb{R})$ of the modular surface. The space of all tilings up to renormalization is isomorphic to the quotient $S A(2, \mathbb{Z}) \backslash S A(2, \mathbb{R})$ of affine measure-preserving maps of the plane by integral ones; this is a fiber bundle with torus fiber over the space of parameters.

Note that, in the general case, the combinatorics and the size of the tiles are completely independent; indeed, one could define a tiling with tiles of size $1, \phi$, and arbitrary Sturmian combinatorics. By inflation, such a tiling will converge to the Fibonacci tiling: in the parameter space, the Fibonacci tiling corresponds to a closed geodesic, and the one we just defined, to an open geodesic that converges to this closed geodesic (see [6] for a detailed study). 
The aim of the present paper is to try to generalize as much of this picture as is possible to higher dimensions.

\section{An example of exduction}

We will now produce a two-dimensional generalization of the Fibonacci tiling, and explain how it can be obtained in several different ways.

We consider the substitution $\sigma: 1 \mapsto 12,2 \mapsto 3,3 \mapsto 1$; it is one of the simplest analogues, on three letters, of the Fibonacci substitution. It has a unique fixed point, starting with $a$, and by taking the closure of the orbit of this fixed point by the shift map $S$, we obtain a symbolic dynamical system $(\Omega, S)$. Note that $\sigma$ is not the usual Rauzy substitution $(1 \mapsto 12,2 \mapsto 13,3 \mapsto 1)$, although it is very close in its form; we have chosen to work out an example which is not already studied in several papers.

It is well known that the dynamical system defined by the Fibonacci substitution is measurably conjugate to the rotation on the circle by the golden number. We ask the question: can we find a similar representation for the dynamical system defined by $\sigma$ ? The answer is yes: this system is conjugate to a rotation on the two-dimensional torus, and the symbolic sequences can be obtained from this rotation by considering itineraries with respect to a suitable partition; however, as we shall see, this partition is not at all trivial.

We give a construction, due to Rauzy (as many of the ideas of this section), to approximate this partition. We wish to represent this system as an exchange of three pieces in the plane; that is, we consider a set $X$, disjoint union of 3 sets $X_{1}, X_{2}, X_{3}$, and three vectors $v_{1}, v_{2}, v_{3}$ (the translation vectors) such that $X$ is also the disjoint union of the sets $X_{i}+v_{i}$; the dynamical system consists in translating by $v_{i}$ each point of $X_{i}$. Such exchanges of three pieces are easy to build, and, under suitable condition, are conjugate to toral rotations. If this system represents the substitution, it is conjugate to the induced map of the exchange of pieces on a subset (this is true for any substitution, since the shift on $\Omega$ is conjugate to its induced map on $\sigma(\Omega)$ ); it is however difficult to build directly a self-induced system. We will instead start from an arbitrary exchange of pieces, and try to "exduce" it, i.e., to build a larger dynamical system, itself also an exchange of pieces, from which the initial one can be induced according to the substitution. This will ensure that, whatever the symbolic dynamics for the initial system, the symbolic dynamics for the exduced system are of the type $\sigma(u)$; by iterating we get symbolic dynamics of the type $\sigma^{n}(u)$. If we can define a limit for this sequence of dynamical systems, it will be conjugate to the substitution.

This can be realized in practice. Consider a dynamical system $(X, T)$, exchange of 3 pieces in an Lshaped domain, (see Figure 3), and enlarge the domain $X$ in the following way: at each step, we consider as a new piece 1 the union of pieces 1 and 3 ( 1 occurs in the image under $\sigma$ of 1 and 3$)$. We translate this union of pieces by the translation vector of the old piece 3 . If this translate of the old piece 1 is disjoint of the previous pieces, we call this new piece 2; the new vector of translation for this new piece 2 is the vector that takes it to the old piece 1 . Finally, old piece 2 is renamed 3 ( 3 occurs in the image of 2 ), which gives the vector of translation. In this way, we obtain a new dynamical system $\left(X^{\prime}, T^{\prime}\right)$; Figure 3 shows the first five steps of the iteration of this procedure.

The following can be easily checked: $(X, T)$ is the induced map of $T^{\prime}$ on $X$; if we consider a point $x \in X$, then we can consider its orbit by $T$, and define a symbolic sequence $u(x)$ by the position of this orbit with respect to the partition of $X$ in three pieces (the old pieces); we can also consider its orbit by $T^{\prime}$, and define another symbolic sequence $v(x)$, using the partition of $X^{\prime}$ by the new pieces. Then we have $v(x)=\sigma(u(x))$, which was the aim of the construction. 
$\begin{array}{ll}3 \\ 1 & 2\end{array}$

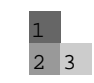

Step 0

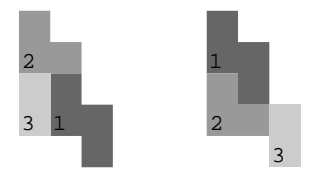

Step 3
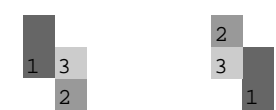

Step 1

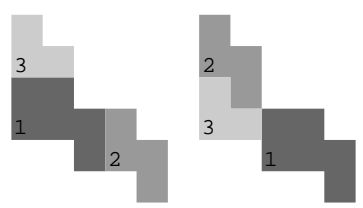

step 4

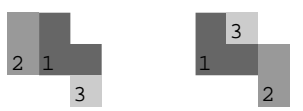

Step 2

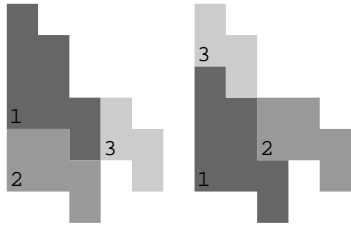

step 5

Fig. 3: Five steps of exduction

If one iterates for a sufficient time, it can be seen experimentally that, after a suitable renormalization, the figures thus obtained converge to an invariant shape. The corresponding formalism was worked out in a series of paper, specially $[23,24,25]$, where it is indeed proved that we can in this way obtain a toral rotation that is conjugate to the system defined by the substitution. The limit domain, which has a fractal boundary in all non-trivial cases, is called the generalized Rauzy fractal of the substitution, following [29].

Much more is true: if we start, not from three squares, but from an hexagon divided into three lozenges (by performing a flip), the same construction produces a tiling by lozenges, that can be seen as the projection of a part of a stepped surface in $\mathbb{R}^{3}$ (see Figure 4); it can be proved that this stepped surface is the best discrete approximation of the invariant contracting plane associated with the matrix of the substitution, thus extending to the higer dimensional case the "cut and project" method of the preceding section.

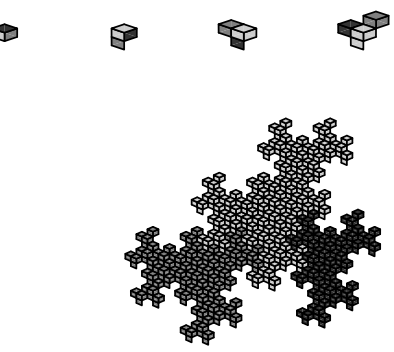

Fig. 4: Exduction and stepped surface

It is a surprising fact that this generalized Rauzy fractal can be recovered in a completely different way, as an atomic surface; it is in this way that it was first explained in the orginal paper in the case of the Rauzy substitution [29]: first build the infinite fixed point of the substitution. Then, associate to this infinite word a broken line in $\mathbb{Z}^{3}$, whose $n$-th vertex is obtained by counting the number of occurences of each letter in the prefix of length $n$ (for example, since the fixed point begins with $1231121 \ldots$, the 
7 th vertex is $(4,2,1))$. It is not difficult to prove that this stepped line stays within bounded distance of the expanding line of the matrix of $\sigma$. If we take the closure of the projection along this direction of the vertices of the stepped surface, we obtain the domain shown in Figure 5; this is the same domain as above. We will give the idea of the proof in the next section.

Let us note that a confusion might occur here in the choice of our terminology (stepped surface and atomic surface): indeed two dual quasicrystals are defined, that is, on one side, the projection on the contracting plane of the stepped surface, and on the other side, the projection, on the expanding line, of the broken line corresponding to the fixed point of the substitution.

The first quasi-crystal has the contracting plane as parallel space, and the expanding line as perpendicular space; it is obtained by the cut and project method, taking as window on the perpendicular space the segement from the origin to the projection of the point $(1,1,1)$. In other words, we slide the unit cube along the contracting plane, and consider all integral points in the slice of space so defined.

The second quasi-crystal is in some way dual to the first; it has the expanding line as parallel space, and the contracting plane as perpendicular space. We can consider the generalized Rauzy fractal as an atomic surface for this second quasi-crystal, while the atomic surface associated with the stepped surface is a segment in the expanding line, and provides a quasi-periodic tiling of the contracting plane by diamonds being the projections of the faces of translates of the unit cube. For more details, see for instance [5]. See also $[35,36]$ for a generation by the cut and project method of such tilings. Let us note that the construction described in [35, 36] corresponds to eigenvectors associated with the substitution $1 \mapsto 123,2 \mapsto 1,3 \mapsto 2$, whose matrix is the tranpose of the matrix of the Rauzy substitution; this explains the difference of some properties between these tilings and the classical Rauzy tilings.

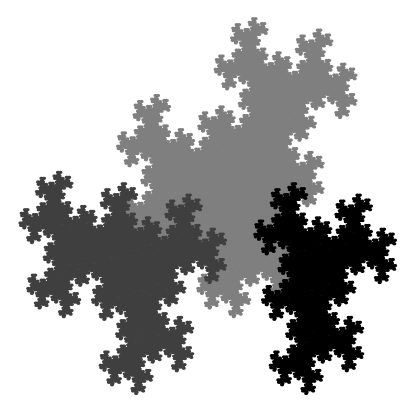

Fig. 5: The atomic surface

This domain is obtained in still other ways; for example, let $\alpha$ be one of the complex roots of the equation $x^{3}-x^{2}-1=0$. Any complex number can be written $z=\sum_{k=-N}^{\infty} \varepsilon_{k} \alpha^{k}$, with $\varepsilon=0$ or 1 , and each 1 is followed by at least two 0 , see [27]. It can be shown that the set of complex number that can be written $z=\sum_{k=0}^{\infty} \varepsilon_{k} \alpha^{k}$ is again the same set (in some sense, these are complex numbers without "integral part" in this numeration system, and the Rauzy fractal plays the role of the unit interval for this numeration system).

The generalized Rauzy fractals can also be used as a basis for the cylinders of a Markov partition [32], and here a result of Bowen [13] tells us that these partitions, in dimension higher than 2, are necessarily complicated, with a fractal boundary, unless they can be split as a direct product of two-dimensional automorphisms. It also appears in the study of the $\beta$-shift (where $\beta$ is the real root of the polynomial $x^{3}-$ 
$x^{2}-1$ ) and its periodic points (see also [22,30]). It is worth remarking that it is much more complicated to study the $\beta$-shift for the real root of the polynomial $x^{3}-x-1$, see Section 6 .

\section{Generalized substitutions}

The aim of this section is to introduce a suitable formalism in order to generate in a systematic way the atomic surface and the stepped surface introduced in the previous section.

\subsection{Atomic surface}

Let $\sigma$ be a substitution defined over the alphabet $\{1,2,3\}$, that is, a non-erasing morphism of the free monoid $\{1,2,3\}^{*}$ endowed with the concatenation. Let $M_{\sigma}$ denote the matrix of incidence of the substitution $\sigma$ : the entry $M_{i, j}$ counts the number of occurrences of the letter $i$ in the word $\sigma(j)$. We will suppose that this matrix is primitive (that is, there exists a power of the matrix that is strictly positive, or equivalently, there exists a power $\sigma^{n}$ of $\sigma$ such that any letter occurs in any word $\sigma^{n}(i)$ ), and, for simplicity, that the matrix is unimodular, that is, has determinant 1 (this will be necessary for Subsection 4.3).

We can then suppose, replacing if necessary $\sigma$ by a power $\sigma^{n}$ and permuting letters, that $\sigma(1)$ begins with 1 , and that there exists an infinite word $u$ starting with 1 such that $\sigma(u)=u$.

We will assume that the substitution $\sigma$ is of Pisot type, that is, $M_{\sigma}$ has a real eigenvalue $\lambda_{1}$, and all other eigenvalues of $M_{\sigma}$ have a modulus strictly less than one (the behaviour of non-Pisot substitutions is much more complicated, and largely unknown). We also assume that the characteristic polynomial of the matrix is irreducible. In that case, there is one expanding direction and a contracting plane $\mathcal{P}$ which is the kernel of the linear form which is the left eigenvector of $M_{\sigma}$ corresponding to $\lambda_{1}$.

One associates with the infinite word $u=\left(u_{n}\right)_{n \in \mathbb{N}}$ a broken line starting from 0 in $\mathbb{Z}^{3}$ and approximating the expanding direction as follows. Let $f:\{1,2,3\}^{*} \rightarrow \mathbb{Z}^{3}$ (abelianization map of the free monoid) be defined by $f(w)=|w|_{1} e_{1}+|w|_{2} e_{2}+|w|_{3} e_{3}$, where $\left(e_{1}, e_{2}, e_{3}\right)$ denotes the canonical basis of $\mathbb{R}^{3}$ and $|w|_{i}$ denotes the number of occurrences of the letter $i$ in the word $w$. We will also use $|w|$ for the length of $w$. Note that for all word $w$, we have $f(\sigma(w))=M_{\sigma} f(w)$. Let us consider the set of points in $\mathbb{Z}^{3}$

$$
\left\{f\left(u_{0} u_{1} \ldots u_{n}\right) ; n \in \mathbb{N}\right\} .
$$

The broken line is defined by joining these points with segments. (Intuitively, we start from the origin, and read the fixed point, moving one step in direction $e_{i}$ if we read letter $i$.) Let $\pi$ denote the projection over the contracting plane $\mathcal{P}$ of the matrix along the expanding direction. We project these points by $\pi$, and define the set $X_{\sigma}$ as the closure of the projection:

$$
X_{\sigma}:=\overline{\left\{\pi\left(f\left(u_{0} \ldots u_{n}\right)\right) ; n \in \mathbb{N}\right\}},
$$

and for $i \in\{1,2,3\}$

$$
X_{\sigma}^{i}:=\overline{\left\{\pi\left(f\left(u_{0} \ldots u_{n}\right)\right) ; n \in \mathbb{N} \text { and } u_{n}=i\right\}} .
$$

The set $X_{\sigma}$ is called the atomic surface associated with $\sigma$.

Proposition 1 - The frequency of the letter $i$ in the infinite word $u$, defined as the limit of the number of occurrences of the letter $i$ in the first $n$ terms of the infinite word, divided by $n$, exists. The vectors $f\left(u_{0} u_{1} \ldots u_{n}\right)$ stay within bounded distance of the expanding direction, which is exactly the direction given by the vector of frequencies of the letters 1,2,3. 
- The set $X_{\sigma}$ is bounded, and hence compact. The translates of the atomic surface by the vectors of the lattice $\mathcal{L}_{0}:=\mathbb{Z} \pi\left(e_{1}-e_{2}\right)+\mathbb{Z} \pi\left(e_{1}-e_{3}\right)$ cover the contracting plane $\mathcal{P}$ :

$$
\cup_{\gamma \in \mathcal{L}_{0}}\left(X_{\sigma}+\gamma\right)=\mathcal{P} .
$$

- The interior of $X_{\sigma}$ is not empty.

Proof. Let us briefly sketch the proof of this result. The matrix $M_{\sigma}$ is by assumption diagonalizable. Let $\left(v_{1}, v_{2}, v_{3}\right)$ be a basis of eigenvectors, associated respectively with the eigenvalues $\lambda_{1}, \lambda_{2}, \lambda_{3}$, where $\lambda_{1}>1 \geq\left|\lambda_{2}\right| \geq\left|\lambda_{3}\right|>0$. By Perron-Frobenius Theorem, $v_{1}$ is a positive vector, and we can suppose that $v_{1}=f_{1} e_{1}+f_{2} e_{2}+f_{3} e_{3}$, with $f_{1}+f_{2}+f_{3}=1$. Let us write $e_{1}=a_{1} v_{1}+a_{2} v_{2}+a_{3} v_{3}$. We have

$$
f\left(\sigma^{k}(1)\right)=M_{\sigma}^{k} e_{1}=a_{1} \lambda_{1}^{k} v_{1}+a_{2} \lambda_{2}^{k} v_{2}+a_{3} \lambda_{3}^{k} v_{3}
$$

which proves that the vectors $f\left(\sigma^{k}(1)\right)$ converge exponentially fast to the expanding line, and their projections on the contracting plane converge to 0 .

This result also holds for prefixes of the fixed point $u$ which are not of the form $\sigma^{k}(1)$. Indeed, it is easy to prove that any prefix $w$ of $u$ can be expanded as:

$$
w=\sigma^{k}\left(w_{k}\right) \sigma^{k-1}\left(w_{k-1}\right) \ldots w_{0},
$$

where the $w_{i}$ belong to a finite set of words. (This corresponds to a "numeration system" on words; there are some admissibility conditions on the possible sequences $\left(w_{i}\right)$, which can be worked out explicitly: they are given by a finite automaton.) It follows easily that $f_{1}, f_{2}, f_{3}$ are equal to the frequencies of the letters, since the convergence in the previous proof is exponentially fast.

This also implies that the set $X_{\sigma}$ is bounded, hence compact: after projection by $\pi$, the component on $v_{1}$ disappears, and there only remains a series that converges exponentially fast. Note that, when $\sigma$ is given, it is possible to give an explicit bound for the diameter of $X_{\sigma}$, using the second eigenvalue and the finite set of words $w_{i}$.

One can check that the vector $v_{1}$ has rationally independent coordinates. Hence $\pi \mathbb{Z}^{3}$ is dense in $\mathcal{P}$. Furthermore,

$$
\left\{f\left(u_{0} u_{1} \ldots u_{n}\right)+\gamma ; n \in \mathbb{N}, \gamma \in \mathcal{L}_{0}\right\}=\left\{(x, y, z) \in \mathbb{Z}^{3}, x+y+z \geq 0\right\} .
$$

Since $X_{\sigma}$ is bounded, we get

$$
\cup_{\gamma \in \mathcal{L}_{0}}\left(X_{\sigma}+\gamma\right)=\mathcal{P}
$$

We deduce from Baire's theorem that the interior of $X_{\sigma}$ is not empty. For more details, see for instance [18]. Note that one can also prove that $X_{\sigma}$ is the closure of its interior.

Remark 1 In combinatorial terms, the previous proof implies that for every letter $i$, Card $\left\{n \in \mathbb{N}, u_{n}=\right.$ $i\}-f_{i} N$ is bounded, with respect to $N$. This is equivalent to the fact that there exists a positive constant $C$ such that the sequence $u$ is $C$-balanced [11], that is, for any two factors $v, w$ of $u$ of the same length, then

$$
\left.|| v\right|_{i}-|w|_{i} \mid \leq C,
$$

for $i=1,2,3$. In dynamical terms, this can be expressed as: the sets $X_{\sigma}^{i}$ are bounded remainder sets with respect to the exchange of pieces we define below [19]. We thus get more generally: 
Proposition 2 The fixed point of a primitive susbtitution of Pisot type is $C$-balanced, for some constant $C$.

Remark 2 We do not know in the general case whether the sets $X_{i}$ can overlap, although in all known examples it has been proved that their intersection has measure 0 . The same question arises for $X_{\sigma}$ and its tranlates by the lattice $\mathcal{L}_{0}$, that is, do the translates of $X_{\sigma}$ tile the contracting plane? These are probably the two most important questions in this field. If one assumes that the substitution is unimodular (the absolute value of the determinant of the matrix equals one), and if one adds an extra combinatorial assumption, that is, the strong coincidence condition, then it can be proved that the sets $X_{\sigma}^{i}$ are disjoint of each other, up to a set of measure zero, and form a partition of $X_{\sigma}$. For more details, see [7]. This implies in particular that the atomic surface has a self-similar structure. Furthermore, the second question (question of tiling) can be also anwered by an algorithmic condition which only depends on the substitution [32].

\subsection{Dynamical approach}

There is a natural shift dynamical system associated with the substitution (take the closure of the shift orbit of the fixed point for the product topology on $\{1,2,3\}^{\mathbb{N}}$ ). The following question is natural. Can we describe in a geometric way, using the atomic surface, the action on one hand of the substitution and on the other hand, of the shift?

Let us assume that the substitution is unimodular and that the sets $X_{\sigma}^{i}$ do not overlap (except on sets of measure zero). As explained in the example of Section 2, we want to describe the shift as an exchange of the pieces $X_{\sigma}^{i}$. This is easy: if we define $X_{\sigma}^{i \prime}:=\overline{\left\{\pi\left(f\left(u_{0} \ldots u_{n}\right)\right) ; n \in \mathbb{N} \text { and } u_{n+1}=i\right\}}$, it is clear that $X_{\sigma}^{i \prime}=X_{\sigma}^{i}-\pi\left(e_{i}\right)$, and that $X_{\sigma}$ is also the disjoint union of the sets $X_{\sigma}^{i^{\prime}}$ (since we project the same points). This was the original proof in [29].

We would like however to formalize the process of exduction discussed previously in Section 3. For that purpose, we will need to introduce a suitable formalism (for more details, see [7, 9]).

Let us start with a geometric description of the substitution $\sigma$. Consider the linear transformation $E_{0}(\sigma)$ defined over $\mathbb{Z}^{3}$ with matrix $M_{\sigma}$. Formally, we can consider a path associated with a finite word as a sum of elements $\left(x, e_{i}\right)$, where $x \in \mathbb{Z}^{d}$. It is then quite natural to consider that the substitution $\sigma$ operates on the set of paths, replacing each segment parallel to $e_{i}$ by a broken line corresponding to the word $\sigma(i)$. We want the image of a connected path to be a connected path; this implies, if we suppose that the point 0 is fixed, that the image of a path joining 0 to $x$ is a path joining 0 to $E_{0}(\sigma)(x)$. This completely defines the image of every segment $\left(x, e_{i}\right)$ (see Figure 6).

For a formal definition, it is more convenient to consider linear combinations of paths with coefficients in $\mathbb{R}$, so as to get a vector space.

Definition 1 Let $\mathcal{F}$ be the vector space of maps from $\mathbb{Z}^{d} \times\{1,2,3\}$ to $\mathbb{R}$ that take value zero except for a finite set. For $x \in \mathbb{Z}^{d}$ and $i \in\{1,2,3\}$, denote by $\left(x, e_{i}\right)$ the element of $\mathcal{F}$ which takes value 1 at $(x, i)$, and 0 elsewhere; the set $\left\{\left(x, e_{i}\right) ; x \in \mathbb{Z}^{d}, i \in\{1,2,3\}\right\}$ is a basis of $\mathcal{F}$, and we define the one-dimensional geometric realization $E_{1}(\sigma)$ of $\sigma$ by

$$
E_{1}(\sigma)(x, i)=\sum_{k=1}^{l_{i}}\left(\left(E_{0}(\sigma)(x)+f\left(P_{k}^{(\mathbf{i})}\right), W_{k}^{(\mathbf{i})}\right)\right.
$$

where we denote $\sigma(i)=W_{1}^{(i)} \ldots W_{k}^{(i)} \ldots W_{l_{i}}^{(i)}=P_{k}^{(i)} W_{k}^{(i)} S_{k}^{(i)}\left(P_{k}^{(i)}\right.$ is the prefix of length $k-1$ of $\left.\sigma(i)\right)$. 

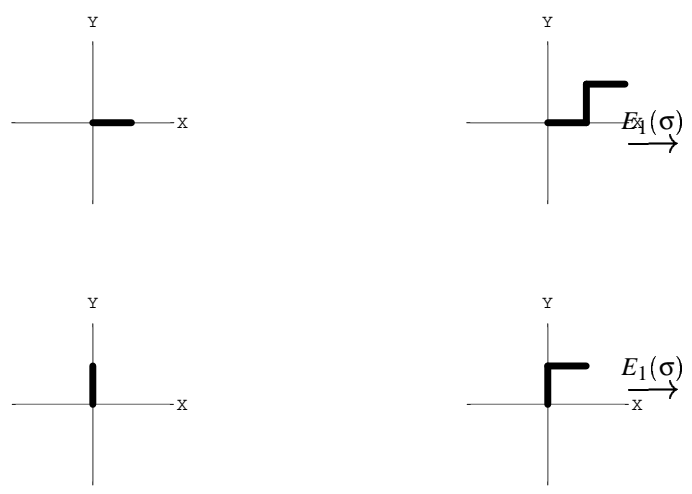

Fig. 6: The linear map associated with $1 \mapsto \mathbf{1 2 1}, \mathbf{2} \mapsto \mathbf{2 1}$

With this definition, we see that the paths $E_{1}(\sigma)^{n}(0,1)$ are increasingly long prefixes of the broken line defined above.

It is possible to generalize this definition to multidimensional extensions of substitutions, to act not only on segments, but also on faces of dimension $k$ [9]. This formalism should be necessary to study substitution which are not of Pisot type.

\subsection{Stepped surface}

We have given a combinatorial meaning to the approximation of the expanding direction, via the broken line associated with $u$. In a dual way, let us now try to approximate the contracting plane.

We will introduce a second type of generalized substitution, which is the dual of the previous one. This map acts over translated faces (by integer vectors) of the unit cube. The purpose of such a device is first to recover in an algorithmic way after a suitable renormalization the atomic surface $X_{\sigma}$ (and thus to describe the exchange of domains on it), and second, to get an explicit way of approximating by successive iterations of the generalized substitution the contracting plane.

We first define the set of basic squares; for $(x, i) \in \mathbb{Z}^{3} \times\{1,2,3\}$, we denote by $\left(x, e_{i}^{*}\right)$ the set $\left\{x+e_{i}+\right.$ $\left.\lambda e_{j}+\mu e_{k} ; 0 \leq \lambda \leq 1,0 \leq \mu \leq 1\right\}$ (where $\{i, j, k\}=\{1,2,3\}$ ).

The idea is to substitute (according to a scheme given by the substitution $\sigma$ ) to a basic square a union of basic squares. When iterating such a device, overlap problems of basic squares may occur. We equip the set of basic squares of a structure of $\mathbb{Z}$-module. Let

$$
\mathcal{F}^{*}:=\left\{\sum_{\left(x, i^{*}\right)} m_{\left(x, i^{*}\right)}\left(x, i^{*}\right), m_{\left(x, i^{*}\right)} \in \mathbb{Z}, m_{\left(x, i^{*}\right)} \text { all zero except a finite set }\right\},
$$

be the set of finite formal sums of weighted faces. Recall that the substitution is unimodular, that is, the matrix $M_{\sigma}$ is invertible as a matrix with integer coefficients. The lattice $\mathbb{Z}^{3}$ acts on $\mathcal{F}^{*}$ in a natural way by translation.

The set $\mathcal{F}^{*}$ can be viewed as the dual space of the space $\mathcal{F}$ defined in the previous section; it is then natural to define the dual map $E_{1}^{*}(\sigma)$, and a straightforward computation leads to the following:

Definition 2 Let $P_{n}^{j}$ denote the prefix of length $n-1$ of $\sigma(j)$, and let $W_{n}^{(j)}$ denote the $j$-th letter of $\sigma(j)$, 
for $j=1,2,3 . E_{1}^{*}(\sigma)$ is the map defined over $\mathcal{F}^{*}$ as follows:

$$
E_{1}^{*}(\sigma)\left(x, i^{*}\right)=\sum_{n, j: W_{n}^{(j)}=i}\left(M_{\sigma}^{-1}\left(x-f\left(P_{n}^{(j)}\right), j^{*}\right),\right.
$$

with the same notation as above for letters and prefixes.

This map is the formalisation of the exduction procedure. More precisely, let $\mathcal{P}^{+}$denote the upper closed half space with boundary $\mathcal{P}$, and define the stepped surface $\mathcal{S}_{\sigma}:=\left\{\left(x, i^{*}\right), x \notin \mathcal{P}^{+},\left(x+e_{i}\right) \in \mathcal{P}^{+}\right\}$ associated with $\mathcal{P}$. Denote by $\mathcal{G}$ the finite linear combinations of elements of $\mathcal{S}_{\sigma}$.

It is easy to prove the following:

Proposition 3 The space $\mathcal{G}$ is invariant by $E_{1}^{*}(\sigma)$. Moreover, each element of $\mathcal{S}_{\sigma}$ is contained in the image, by $E_{1}^{*}(\sigma)$, of exactly one element of $\mathcal{S}_{\sigma}$.

Hence, after iteration of the generalized substitution $E_{1}^{*}(\sigma)$, starting from lower faces of the unit cube at the origin (these always belong to $\left.\mathcal{S}_{\sigma}\right)\left(-e_{1}, 1^{*}\right)+\left(-e_{2}, 2^{*}\right)+\left(-e_{3}, 3^{*}\right)$, one can generate arbitrarily large parts of the stepped surface associated with the contracting plane $\mathcal{P}$.

After normalization of the projection of these arbitrarily large parts of the stepped surface, the sequence of sets

$$
M_{\sigma}^{n}\left(\pi\left(\left(E_{1}^{*}(\sigma)\right)^{n}\left[\left(-e_{1}, 1^{*}\right)+\left(-e_{2}, 2^{*}\right)+\left(-e_{3}, 3^{*}\right)\right]\right)\right.
$$

can be seen to converge towards the set $-X_{\sigma}$, in the Hausdorff topology. For more details, see [7].

Remark 3 The map $E_{1}^{*}(\sigma)$ is easy to implement effectively on computer; this is how Figure 4 was produced. This allows computations which are quite difficult by hand: it is not so easy to continue the exduction procedure shown in Section 3!

\subsection{The problem of tiling by the atomic surface}

We have worked here in order to clarify the situation with a substitution over a three-letter alphabet. All the results extend in a natural way to large size alphabets, provided we consider Pisot primitive unimodular substitutions, with the strong coicidence property. We have seen that the translates by the vectors of the lattice of the atomic surface cover the contracting plane. The following question is thus natural. Do we get a tiling of this plane?

Instead of working directly with the atomic surface, one can consider the Hausdorff limit of the renormalized patches of the stepped surface built by $E_{1}^{*}(\sigma)$. It is easy to prove directly that these renormalized patches tile the contracting plane, using the lattice $\mathcal{L}_{0}$. The problem is that the limit could have a larger measure, and overlap with its translates. This could happen if the boundaries of the patches become very wild, and converge to a set of dimension 2.

This question is still an open problem and can be formulated under several different forms. Let us note that we do not know any example which does not verify the previous assumption. One can only prove, in the general case, that the fibers of the covering by the translates of the atomic surface are finite almost everywhere. In ergodic terms, this can be expressed as:

Theorem 1 Any unimodular Pisot substitutive dynamical system over a d-letter alphabet which satisfies the strong coincidence condition admits as a continuous factor an explicit irrational translation on the torus $\mathbb{T}^{d-1}$, the fibers being finite almost everywhere. 
The question of the measure-theoretical isomorphism with the rotation factor is equivalent to the problem of tiling. Let us note that A. Siegel has produced in [32] an explicit and effective combinatorial condition (based on a clever graph study) for the atomic surface associated with a unimodular Pisot susbtitution over $d$ letters to generate a regular tiling of $\mathbb{R}^{d-1}$.

A sufficient condition for the tiling is the following: there exists a set with nonempty interior which is ultimately included in the sets of the sequence of approximations we build for the atomic surface, i.e., which is included in $M_{\sigma}^{n}\left(\pi\left(\left(E_{1}^{*}(\sigma)\right)^{n}\left[\left(-e_{1}, 1^{*}\right)+\left(-e_{2}, 2^{*}\right)+\left(-e_{3}, 3^{*}\right)\right]\right)\right.$ for $n$ large enough.

\section{Generalized continued fractions}

We have explained in the previous section how one can build the stepped surface associated with the contracting plane of a certain type of matrices, and how to build the atomic surface associated with the expanding direction.

Note that we are restricted to some very particular planes and lines: this can only work for some planes and lines whose coordinates are algebraic, since they are given by eigenvectors of some integral matrices. This is reminiscent of Section 2, where we first gave approximations for lines with some quadratic slopes. As we did in Subsection 2.3, we will give a procedure, using generalized continued fractions, that allows us to build a stepped surface associated with a plane and an atomic surface associated with a direction.

The choice of the generalized continued fraction algorithm is arbitrary; its influence on the atomic surface is at the moment unclear. We will not give technical details on which algorithm we use, but just sketch the main lines (in the papers [24, 25, 5], the usual algorithms are Jacobi-Perron and Brun (also called modified Jacobi-Perron, or generalized Gauss) algorithms).

\subsection{Generation of the stepped surface}

We consider a plane $\mathcal{P}_{\alpha, \beta}$ normal to a vector $(1, \alpha, \beta)$, and we approximate this vector by a continued fraction algorithm. The dynamical system associated with this algorithm (generalized Gauss map) produces a sequence of vectors $\left(1, \alpha_{n}, \beta_{n}\right)$, associated normal planes $\mathcal{P}_{n}$, and a sequence of matrices $M_{n}$ with positive coefficients, such that $M_{n}^{-1}$ sends plane $\mathcal{P}_{n}$ to plane $\mathcal{P}_{n-1}$.

We can associate to this sequence of matrices in a canonical way a sequence of substitutions $\sigma_{n}$, such that the dual one-dimensional extension of the substitution, $E_{1}^{*}\left(\sigma_{n}\right)$, sends the stepped surface of the plane $\mathcal{P}_{n}$ to the stepped surface of the plane $\mathcal{P}_{n-1}$.

It is clear that, for any plane orthogonal to a strictly positive vector, the union $\mathcal{U}:=\sum_{i=1,2,3}\left(-e_{i}, i^{*}\right)$ of the three faces at the origin, defined above, belongs to the stepped surface; hence, the sets obtained by iterating the dual substitutions $E_{1}^{*}\left(\sigma_{1}\right) E_{1}^{*}\left(\sigma_{2}\right) \ldots E_{1}^{*}\left(\sigma_{n}\right)(\mathcal{U})$ give us arbitrarily large parts of the stepped surface.

This formula is not completely trivial: at each step, we must consider $\mathcal{U}$ as a small part of a different stepped surfaces; the composition of the generalized substitutions sends this set to the stepped surface of the initial plane $\mathcal{P}_{0}=\mathcal{P}_{\alpha, \beta}$.

Remark that we do not always generate in this way all of the stepped surface: there are, for example, special cases of the Jacobi-Perron algorithm where it is possible to prove that, although we can build arbitrarily large parts of the stepped surface (enough to understand completely its combinatorics, and to see every finite pattern that could occur), some parts of the stepped surface can never be attained in this way (see $[25,5])$. Nevertheless, these cases might be considered as exceptional. 


\subsection{Generation of the atomic surface}

One can also generate the atomic surface associated with a direction: consider a vector $(1, \gamma, \delta)$, and an arbitrary plane $\mathcal{P}_{0}$ (orthogonal to a positive vector).

One can then approximate the vector $(1, \gamma, \delta)$ by a continued fraction algorithm; we again recover a sequence of matrices, and of substitutions $\sigma_{n}$, now associated with this vector $(1, \gamma, \delta)$. One can now consider the direct one-dimensional extension of the substitutions $E_{1}\left(\sigma_{n}\right)$. By composition, one checks that the finite broken lines $E_{1}\left(\sigma_{1}\right) E_{1}\left(\sigma_{2}\right) \ldots E_{1}\left(\sigma_{n}\right)\left(0, e_{i}\right)$ approximate the direction of $(1, \gamma, \delta)$, and, under suitable hypothesis (for example, if $\sigma_{n}(1)$ always begins with 1 ), one obtains a limit broken line approximating this direction.

It is not clear that this broken line stays within bounded distance of the line generated by $(1, \gamma, \delta)$; one would like to imitate the argument given in the case of a constant substitution, but this relies on the fact that the endpoints of the successive finite broken lines we obtain converge exponentially fast to the line. This is really a probleme of Diophantine approximation.

One important argument to achieve this is the following Diophantine approximation theorem [20]. For almost every $(\gamma, \delta) \in[0,1)^{2}$, the sequence of simultaneous approximations of $(\gamma, \delta)\left(\frac{p_{n}}{q_{n}}, \frac{r_{n}}{q_{n}}\right)$, produced by the modified Jacobi-Perron algorithm satisfy:

$$
\exists \varepsilon>0,\left\|q_{n}(\gamma, \delta)-\left(p_{n}, r_{n}\right)\right\|<\frac{1}{\left(q_{n}\right)^{\varepsilon}}, \text { for } n \text { large enough. }
$$

Hence, for almost every $\gamma, \delta$, the broken line stays within bounded distance of the line generated by $(1, \gamma, \delta)$; by projecting the vertices of this broken line on the plane $\mathcal{P}$, and taking the closure, one gets again an atomic surface depending on this vector.

It is also possible to approximate this atomic surface by finite unions of parallelograms, using renormalization of the dual one-dimensional extension of the substitutions $\sigma_{n}$; we now consider the vectors $\left(1, \gamma_{n}, \delta_{n}\right)$, the matrices $M_{n}$, and the planes $\mathcal{P}_{n}$ images of the initial plane $\mathcal{P}$, and we denote by $\pi_{n}$ the projection on $\mathcal{P}_{n}$ along $\left(1, \gamma_{n}, \delta_{n}\right)$.

It is now possible to prove that, under the same hypothesis of Diophantine approximation, the sequence of sets $M_{1} \ldots M_{n} \pi_{n} E_{1}^{*}\left(\sigma_{n}\right) \ldots E_{1}^{*}\left(\sigma_{1}\right)(\mathcal{U})$ converge in the Hausdorff sense to a compact set, which is the same atomic surface we obtained by projection.

Remark that the composition of dual substitutions is done now on the opposite order of what we did in the previous subsection. The meaning is the following: starting from $\mathcal{U}$, considered as a part of the stepped surface of $\mathcal{P}$, we obtain a large part of the stepped surface of the plane $\mathcal{P}_{n}$; we then project this stepped surface on $\mathcal{P}_{n}$ along $\left(1, \gamma_{n}, \delta_{n}\right)$, and renormalize back to the initial plane using the product of the matrices $M_{n}$. For more details, see [25, 21, 5].

Let us note that this approach has to be compared with the notion of "approximant structures" in the context of quasicrystals (see for instance $[35,36]$ ).

\subsection{Natural extensions}

One interest of the modified Jacobi-Perron algorithm of is that it admits a simple realization of its natural extension [8]; roughly speaking, one can define a one-to-one map $\bar{T}$ on $([0,1) \times[0,1))^{2}$ such that its positive iterations on the parameter $(\alpha, \beta, \gamma, \delta)$ produce the continued fraction expansion according to the modified Jacobi-Perron algorithm of $(\alpha, \beta)$, whereas its negative iterations produce the continued fraction expansion of $(\gamma, \delta)$. We thus get a biinfinite sequence of digits $\left(a_{n}, \varepsilon_{n}\right)_{n \in \mathbb{Z}}$. In that case, we can do 
simultaneously what we did in the previous two sections. We obtain a biinfinite sequence of matrices $M_{n}$ and of substitutions $\sigma_{n}$, indexed by $\mathbb{Z}$; using the positive part of this sequence, associated with $(1, \alpha, \beta)$, we compute the stepped surface of the plane $\mathcal{P}_{\alpha, \beta}$, and using the negative part, we obtain in that plane, under suitable Diophantine hypothesis (always satisfied, for example, in the case of periodic expansion), an atomic surface associated with the vector $(1, \gamma, \delta)$. This surface is naturally decomposed into 3 pieces, and we may combine both to obtain a tiling, with the same combinatorics as the stepped surface, and tiles given by the pieces of the atomic surface.

In the periodic case, this is a self-similar tiling; in the general case, it is an "hetero-similar" tiling, that can be inflated or deflated to other tilings of the same type, obtained by shifting the biinfinite continued fraction expansion. We thus obtain generalized Sturmian tilings in the sense of $[35,36]$.

\subsection{Additional remarks}

There are known examples where the atomic surface does not exist, because the corresponding broken line does not stay within bounded distance of the initial line; one such example is the case of sequences of complexity $2 n+1$, which are obtained by a particular continued fraction algorithm; a recent paper ([14]) shows that some of these sequences are not balanced, which is equivalent to the fact that the projection of the corresponding broken line is not bounded.

We have always considered here what happens when we project on the plane along the line. There is a dual viewpoint, corresponding to $\mathbb{Z}^{2}$-actions on the circle instead of rotations on the torus: one could project on the line along the plane. In this way, one can also build a tiling of the line, and a trivial atomic surface, that is, an interval. See for instance, [5, 34].

In some cases, we can use the atomic surface on the plane and the atomic surface on the line to produce "generalized Markov partitions", which are true Markov partitions for some toral automorphism in the periodic case, and which can be considered as Markov partitions for the composition of a biinfinite sequences of automorphisms (the matrices $M_{n}$ given by the continued fraction expansion) in the general case.

\section{An Application to tilings issued from $\beta$-expansions}

Let $\beta$ be a Pisot number. Consider the numeration system associated with $\beta$. It is known since Thurston [33] and Akiyama [2,3] that one can associate with this numeration system a finite set of TA-prototiles with fractal boundary $\mathcal{T}$ and a TA-tiling $T$. Consider in particular (Figure 7 ) the Pisot unit $\beta$ whose minimal polynomial is $X^{3}-X-1$ and whose characteristic polynomial (for the $\beta$-expansion) is

$$
X^{5}-X^{4}-1=\left(X^{2}-X+1\right)\left(X^{3}-X-1\right) .
$$

It would be natural to try to recover the tiling using the substitution $a \mapsto b, b \mapsto a c, c \mapsto a$, whose characteristic polynomial is the minimal polynomial given above; unfortunately, this does not work well (one gets a quite wild tiling, with disconnected tiles).

In order to recover this tiling using the formalism with generalized substitutions, we need to introduce a substitution $\sigma$ on five letters [17], the characteristic polynomial of which is given by the previous polynomial of degree 5 .

Let $X$ be the atomic surface associated with the fixed point of the substitution by projection on the contracting place of the corresponding broken line; then the atomic surface $X$ coincides with the tile 


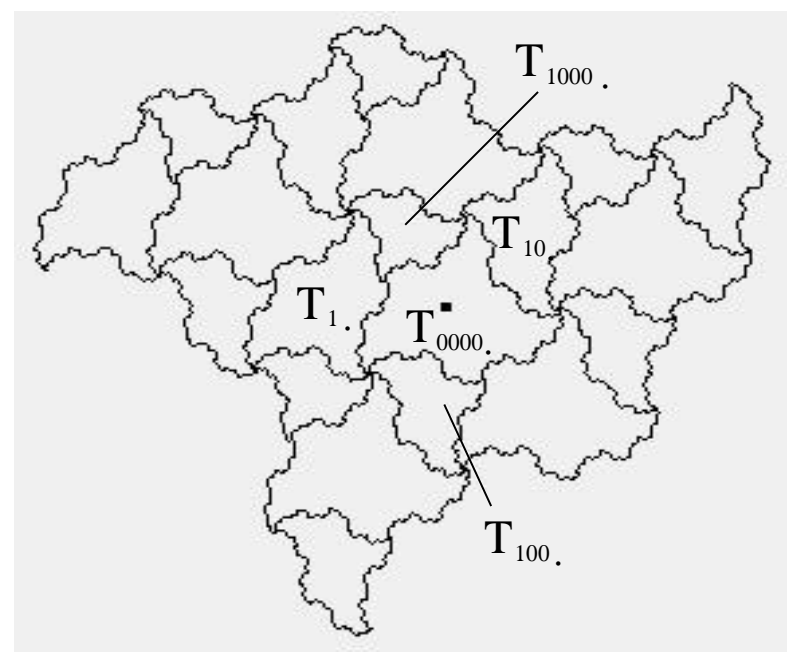

Fig. 7: The TA-tile associated with $\beta$

$\mathcal{T}$. The difference with the previous sections is that the projection is here on a two-dimensional plane corresponding to the conjugates of $\beta$, along a three-dimensional space.

It turns out that this atomic surface can also be generated by a renormalization process, using a generalized substitution acting on a special set of tiles. In fact, using the substitution $\sigma$, we introduce a tiling substitution $\tau^{*}$ which produces a nonperiodic but quasiperiodic tiling $T$ with five protiles (see Figure 6). One can furthermore endow the atomic surface with an exchange of pieces (see Figure 6,6). The meaning of this exchange of pieces is at that moment unclear. More details can be found in [17].

\section{Acknowledgements}

We would like to thank R. Mosseri for his helpful comments on the connections with quasicrystals.

\section{References}

[1] R. L. Adler and B. Weiss Similarity of automorphisms of the torus, Memoirs of the American Mathematical Society 98 (1970), 1-43.

[2] S. Akiyama Pisot numbers and greedy algorithm, "Number Theory, Diophantine Computational and Algebraic Aspects”, ed. by K. Györy, A. Pethö and V. T. Sós, de Gruyter (1998), 9-21.

[3] S. Akiyama Self affine tiling and Pisot numeration system, "Number Theory and its Applications", ed. by K. Györy and S. Kanemitsu, Kluwer (1999), 7-17.

[4] P. Arnoux Chaos from order, a worked out example, in Complex Systems, E. Goles and S. Martinez (Eds), Kluwer Academic Publ, (2001), 1-67. 

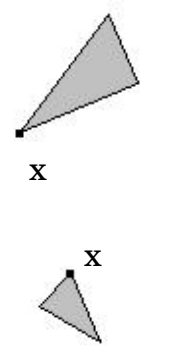
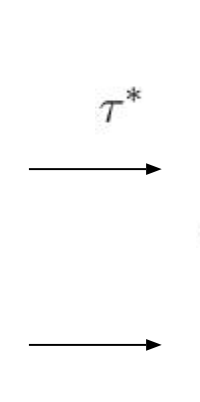
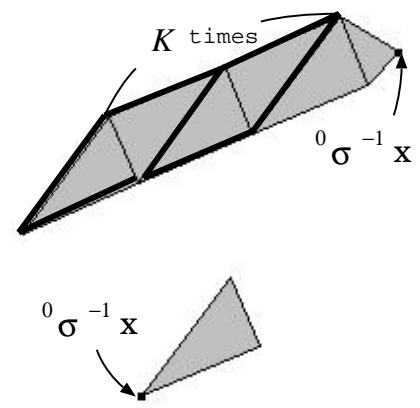
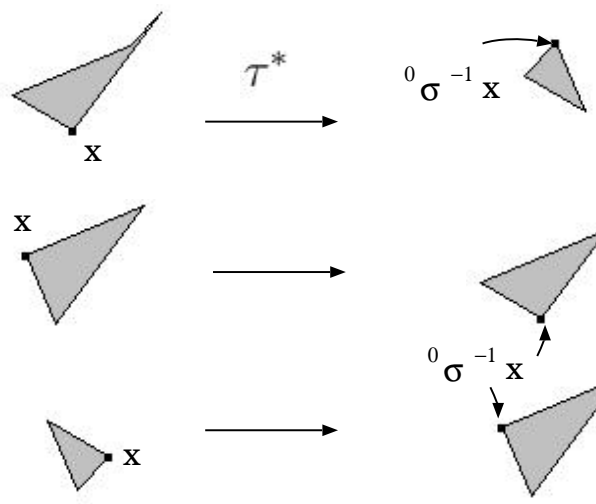

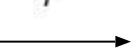

$\sigma$

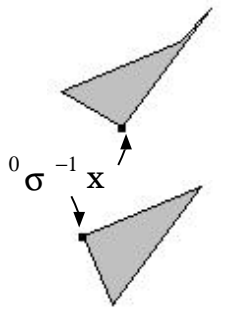

Fig. 8: The substitution $\tau^{*}$

[5] P. Arnoux, V. Berthé, S. Ito Discrete planes, $\mathbb{Z}^{2}$-action, Jacobi Perron algorithms and substitutions, prepublication 2001.

[6] P. Arnoux, A. Fisher The scenery flow for geometric structures on the torus: the linear setting, preprint submitted.

[7] P. Arnoux, S. Ito Pisot substitutions and Rauzy fractals, Bull. Belg. Math. Soc., to appear.

[8] P. Arnoux, A. Nogueira Mesures de Gauss pour des algorithmes de fractions continues multidimensionnelles, Ann. Sci. École Norm. Sup. 26 (1993), 645-664.

[9] P. Arnoux, S. Ito, Y. Sano Higher dimensional extensions of substitutions and their dual maps, J. Anal. Math., to appear.

[10] J. Berstel Recent results in Sturmian words, Developments in Language Theory II (Dassow, Rozenberg, Salomaa eds) World Scientific (1996), 13-24.

[11] V. Berthé, R. Tijdeman Balance properties of multi-dimensional words, Theoret. Comput. Sci., to appear.

[12] V. Berthé, L. Vuillon Tilings and rotations on the torus: a two-dimensional generalization of Sturmian sequences, Discrete Math. 223 (2000), 27-53.

[13] R. Bowen Markov partitions are not smooth, Proceedings of the American Mathematical Society 71 (1978), 130-132.

[14] J. Cassaigne, S. Ferenczi, L. Zamboni Imbalances in Arnoux-Rauzy sequences, Ann. Inst. Fourier 50 (2000), 1265-1276.

[15] N.G. deBruijn Sequences of zeros and ones generated by special production rules, Kon. Nederl. Akad. Wetensch. Proc. Ser. A, 84 (1981), 38-52. 


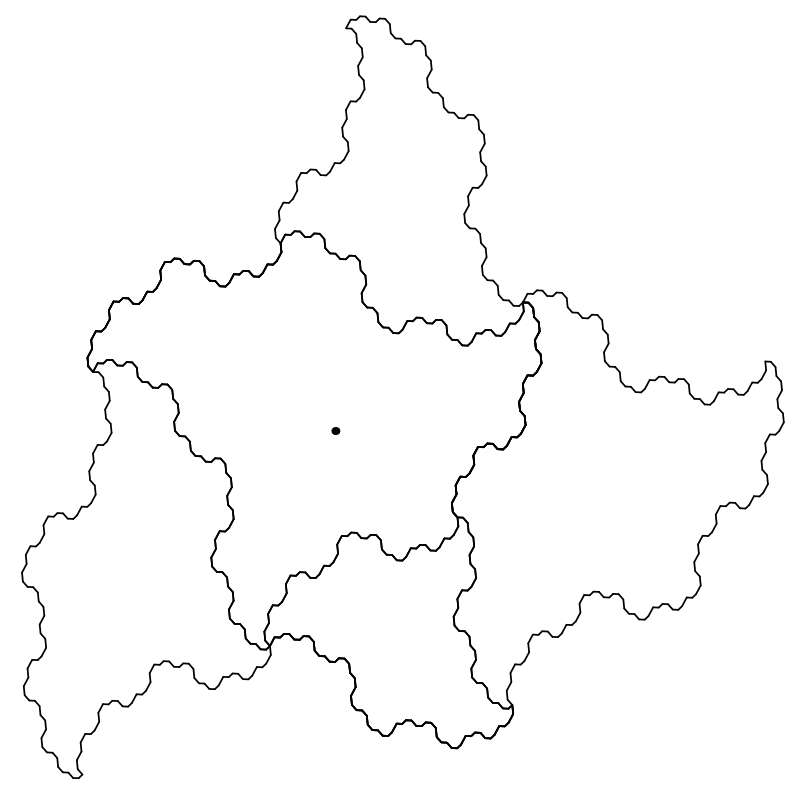

Fig. 9: The exchange of pieces: the domain

[16] N.G. deBruijn Updown generation of Beatty sequences, Kon. Nederl. Akad. Wetensch. Proc. Ser. A, 92 (1989), 385-407.

[17] H. Ei, S. Ito Tilings from characteristic polynomials of $\beta$-expansions, preprint 2001.

[18] D. J. Feng, M. Furukado, S. Ito, J. Wu Pisot substitutions and the Hausdorff dimension of atomic surfaces, preprint 2000.

[19] S. Ferenczi Bounded remainder sets, Acta Arith. 61 (1992), 319-326.

[20] T. Fujita, S. Ito, M. Keane, M. Ohtsuki On almost everywhere exponential convergence of the modified Jacobi-Perron algorithm: a corrected proof, Ergodic Theory Dynam. Systems 16 (1996), 13451352.

[21] S. Ito Fractal domains of quasi-periodic motions on $\mathbb{T}^{2}$, “Algorithms, Fractals, and Dynamics", ed. by Y. Takahashi, Plenum Press, New York (1995), 95-99.

[22] S. Ito On periodic expansions of cubic numbers and Rauzy fractals, "Dynamical systems, From Crystal to Chaos, Proceedings of the conference in honor of Gérard Rauzy on his 60th birthday", ed. by J.-M. Gambaudo, P. Hubert, P. Tisseur, S. Vaienti, World Scientific (2000), 144-164.

[23] S. Ito, M. Kimura On Rauzy fractal, Japan J. Indust. Appl. Math. 8 (1991), 461-486. 


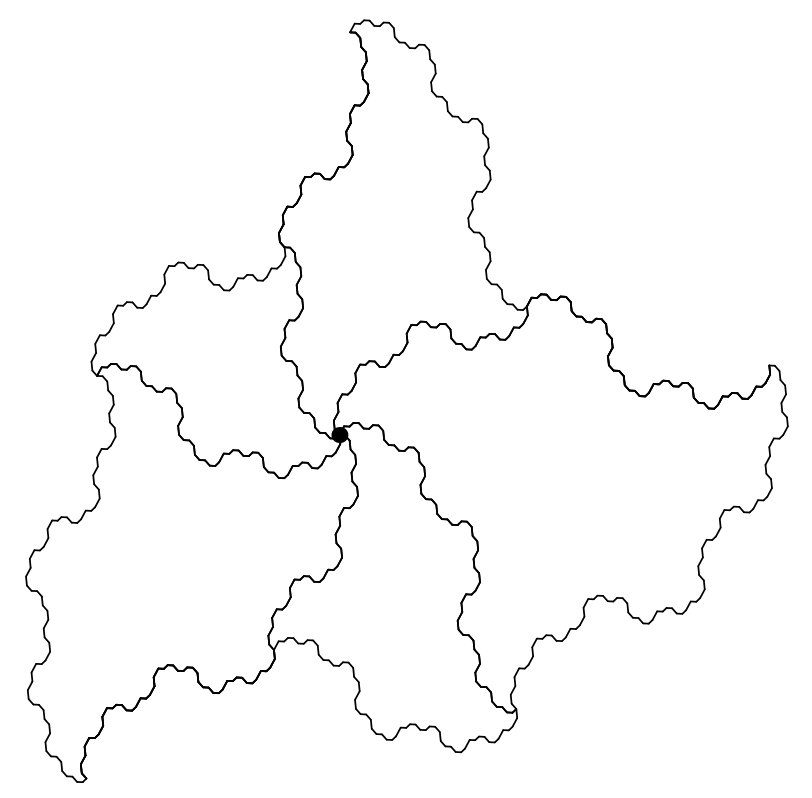

Fig. 10: The exchange of pieces: the image

[24] S. Ito, M. Ohtsuki Modified Jacobi-Perron algorithm and generating Markov partitions for special hyperbolic toral automorphisms, Tokyo J. Math. 16 (1993), 441-472.

[25] S. Ito, M. Ohtsuki Parallelogram tilings and Jacobi-Perron algorithm, Tokyo J. Math. 17 (1994), 33-58.

[26] M. Lothaire Algebraic Combinatorics on Words, Chapitre 2: Sturmian words, par J. Berstel et P. Séébold, to appear.

[27] A. Messaoudi, Frontière du fractal de Rauzy et système de numération complexe, Acta Arith. 95 (2000) 195-224

[28] M. Morse, G. A. Hedlund Symbolic dynamics II: Sturmian trajectories, Amer. J. Math. 62 (1940), $1-42$.

[29] G. Rauzy Nombres algébriques et substitutions, Bull. Soc. math. France 110 (1982), 147-178.

[30] Y. Sano On purely periodic beta-expansions of Pisot numbers, J. Anal. Math., to appear.

[31] M. Senechal Quasicrystals and geometry, Cambridge University Press, Cambridge, 1995.

[32] A. Siegel Représentations géométrique, combinatoire et arithmétique des systèmes substitutifs de type Pisot, Thèse, Université Aix-Marseille II, 2000. 
[33] W. P. Thurston Groups, Tilings and Finite state automata, AMS Colloquium lectures, 1989.

[34] J.-L. Verger-Gaugry, J.-P. Gazeau Geometric study of the set $\mathbb{Z}_{\beta}$ of beta-integers with $\beta$ a Perron number, a $\beta$-number and a Pisot number and mathematical quasicrystals, preprint 2001.

[35] J. Vidal, R. Mosseri Generalized Rauzy tilings: construction and electronic propertie, Materials Science and Engineering A 294-296 (2000), 572-575.

[36] J. Vidal, R. Mosseri Generalized quasiperiodic Rauzy tilings, J. of Physics A, Math. and Gen., may 2001 issue.

[37] L. Vuillon Combinatoire des motifs d'une suite sturmienne bidimensionnelle, Theoret. Comput. Sci. 209 (1998), 261-285.

[38] E. Zeckendorf Représentation des nombres naturels par une somme de nombres de Fibonacci ou de nombres de Lucas, Bull. Soc. Roy. Sci. Liège 41 (1972), 179-182. 